\title{
Better Outcomes with Minimally Invasive Thyroidectomy than Conventional Thyroidectomy
}

Nilda Sütay Süslü ${ }^{1}$, [MD]

ORCID: 0000-0001-9901-3044

Cagri Kulekci ${ }^{2, *},[\mathrm{MD}]$

ORCID: 0000-0002-4981-7711

Selcuk Dagdelen ${ }^{3},[\mathrm{MD}]$

ORCID: 0000-0002-0513-1750

Nafiye Yildiz ${ }^{4}$, [MD]

ORCID: 0000-0001-6652-0605

Tomris Erbas ${ }^{3}$, [MD]

ORCID: 0000-0003-1377-9394

'Department of Otorhinolaryngology, Hacettepe University, Ankara, Turkey

${ }^{2}$ Otorhinolaryngology, Akyurt State Hospital, Ankara, Turkey

${ }^{3}$ Department of Endocrinology and Metabolism, Hacettepe University, Ankara, Turkey

${ }^{4}$ Department of Endocrinology, Hitit University Erol Olçok Education and Research Hospital,Çorum, Turkey

Corresponding Author: Cagri Kulekci Otolaryngology Akyurt State HospitalAnkara, 06750, Turkey. Tel: +903123054224,Fax: +903123113500 e-mail: cagrikulekci@gmail.com

\section{we) ABSTRACT Cen}

Objective: Minimally invasive thyroidectomy is a relatively new technique used in selected patients with success. Aim of this study was to evaluate our results from conventional and minimally invasive thyroidectomy and compare their outcomes.

Materials and Methods: 137 patients undergone conventional and minimally invasive thyroidectomy (video-assisted and open technique with mini-cervicotomy) were reviewed. Demographic data, blood loss, operative time, postoperative pain, cosmetic outcome and complications were examined and compared.

Results: Minimally invasive group consists of 53 cases (39\%) and conventional group is composed of 84 cases (61\%). No conversion from minimally invasive to conventional techniques was observed. In minimally invasive group, 25 patients (47\%) had differentiated thyroid carcinoma and 28 patients (53\%) had benign pathologies. Postoperative complications include transient hypocalcemia in 5 patients (3.6\%) (4 in conventional, 1 in minimally invasive group), permanent hypocalcemia in 4 patients (3\%) (3 in conventional, 1 in minimally invasive group), unilateral vocal cord paralysis in 2 patients (2\%) (1 in conventional, 1 in minimally invasive group). Operative time was similar in both groups; however, blood loss during surgery, postoperative pain was significantly lower in minimally invasive group.

Conclusion: Minimally invasive techniques could be implemented on selected carcinoma patients and results in equal safety and less surgical morbidity.

Keywords: Minimally invasive thyroidectomy, thyroid cancer, MIVAT

Received: 28 October 2019, Accepted:30 December 2019,

Published online: 31 December 2019

\section{INTRODUCTION}

The modern surgery relies not only on eradicating disease but also preserving uninvolved tissues, respecting surroundings. Minimally invasive techniques constitute many standard surgical approaches today; ranging from abdominal, pelvic to orthopedic operations. They almost always include smaller incisions, less tissue disruption; thus result in lower surgical morbidity, less postoperative pain and better cosmetic and functional results.

Minimally invasive thyroidectomy was introduced in 1990 s as a diagnostic approach to small thyroid nodules and then utilized for total thyroidectomy and total thyroidectomy with central neck dissection.
For the improved cosmetic outcomes and minimizing postoperative pain, Micolli et al were among the first to introduce minimally invasive video assisted thyroidectomy (MIVAT) in 1999[1]. It relies on 1.5 $\mathrm{cm}$ mini-cervicotomy and video assisted visualization of thyroid vessels and laryngeal nerves. Further studies showed that minimally invasive techniques offer smaller scars, less postoperative pain and do not involve more surgical complications than conventional procedures [2-5].

Although there are controversies about the definition, in current literature, minimally invasive 
thyroidectomy encompasses transcervical approaches with incisions smaller than $3 \mathrm{~cm}$, endoscopic or non-endoscopic; or extracervical approaches from axilla, breast, anterior chest and oral vestibule [6-8].

The aim of this paper is to contribute our experience to the literature and compare our results from both conventional and minimally invasive thyroid surgeries.

\section{MATERIALS and METHODS}

Following the ethical committee approval, between 2012 and 2016, all patients who underwent thyroidectomies were reviewed. Thyroidectomy cases that were part of laryngeal, hypopharyngeal and esophageal carcinoma operations and patients without appropriate follow up data were excluded. 137 patients were found eligible for the study. Thyroidectomies performed for nodular goiter, multinodular goiter and differentiated thyroid carcinomas are included in the study. Two groups were constructed as conventional surgery and minimally invasive groups. Conventional surgery group include conventional open approach; whereas minimally invasive group include mini-cervicotomy with less than $3 \mathrm{~cm}$ with or without video assistances. Central compartment dissection was done in indicated cases regardless of the group.

Preoperative investigation included thyroid function tests, thyroid ultrasound and both thyroid nodules and total thyroid volume were calculated. Minimally invasive thyroidectomy indications were as follows: a) thyroid nodules less than $3 \mathrm{~cm}$, b) thyroid volume less than $35 \mathrm{ml}, \mathrm{c}$ ) no previous neck surgery or radiation, d) small differentiated thyroid carcinomas with or without central lymph node metastasis. Patients fulfilling minimally invasive thyroid surgery and conventional group were informed about the procedure and associated surgical risks and complications.

Surgery

All the surgeries were performed by the two academic staff specialized in head and neck surgery. Minimally invasive thyroid operations include thyroidectomies with mini-cervicotomy, or mini-cervicotomy with video assistance. All other approaches were classified as conventional thyroidectomy. Minimally invasive surgery is performed as follows: the incision is $2 \mathrm{~cm}$ above the sternal notch and $1.5 \mathrm{~cm}$ to $2 \mathrm{~cm}$ in diameter. Following skin incision, strap muscles are divided in the midline and retracted to develop a plane between thyroid gland and muscles. 30-degree $4 \mathrm{~mm}$ endoscope is introduced in the operative field and superior and inferior pedicles are identified and parathyroid glands and inferior laryngeal nerves visualized and preserved. After performing the necessary excision, straps are approximated and appropriate drain is placed. Following subdermal and epidermal sutures, pressure dressing is applied.

Postoperative Follow Up

Operative time and blood loss was recorded and each surgery was registered in detail. If vessel sealing device was used, it was recorded as well. All thyroidectomy patients were hospitalized postoperatively. The following morning, after removing the dressing, the patients' pain status was measured by Wong-Baker FACES ${ }^{\circledR}$ Pain Scale: ranging from 0 (no pain) to 10 (unbearable pain) with respected facial figures by the numbers. Following appropriate medical treatment, all patients were discharged with analgesic, and calcium replacement therapy if hypocalcemic. Patients were advised to administer back to the hospital if they exhibit hypocalcemia symptoms.

Vocal cord mobility was checked in the first postoperative day via fiberoptic laryngoscopy. Cosmetic outcome was evaluated after 1 month. Visual Analogue Score (VAS) was used to scale cosmetic satisfaction; ranging from $0-10$; 0 being totally unsatisfied and 10 being the most satisfactory result. Like pain scale, cosmetic scale also had sad and smiling faces accompanying numbers.

All patients showing vocal cord dysfunction were followed up and permanent vocal cord paralysis is diagnosed as the persistence of vocal cord paralysis at 6 months postoperatively. Permanent hypocalcemia is defined when the patient depends on replacement therapy after 6 months from the surgery. Hypocalcemia that resolved before that period is considered transient.

Statistics

Operation type, operative time, blood loss, LigaSure $^{\mathrm{TM}}$ (Medtronic, Minneapolis, MN) usage, drain usage, postoperative pain, complications, cosmetic outcome and pathological diagnosis were analyzed and compared between the two groups using SPSS 20 (SPSS Inc, Chicago, III). For blood loss, operative time, postoperative pain and cosmetic outcome differences between the conventional and minimally invasive group were examined using $t$ test, drain usage difference was measured by 
Better outcomes with minimally invasive thyroidectomy chi-square test. $p$ values less than 0,05 were considered statistically significant.

\section{RESULTS}

Ninety-four patients had total thyroidectomy and 43 patients had lobectomy. Thirty-seven of 94 patients

(39\%) had minimally invasive total thyroidectomy, and 57 patients (61\%) underwent conventional total thyroidectomy. Of the 43 patients who had lobectomy, 16 (59\%) underwent minimally invasive surgery, and other 27 (41\%) underwent conventional lobectomy. Baseline characteristics of the study group are given in the Table 1.

Table 1. Baseline characteristics and postoperative pathology of the groups. (CT: conventional thyroidectomy, MIT: minimally invasive thyroidectomy)

\begin{tabular}{|l|c|c|c|c|}
\hline & & CT group & MIT group & p value \\
\hline Male/ Female & & $28 / 56$ & $15 / 38$ & 0.54 \\
\hline Age (mean) & & $48 \pm 15$ & $45 \pm 12$ & 0.16 \\
\hline Operation & Total Thyroidectomy & 57 & 37 & 0.81 \\
\hline \multirow{2}{*}{ Pathology } & Lobectomy & 27 & 16 & \\
& Nodular Goiter & 36 & 25 & 0.63 \\
\hline
\end{tabular}

CT: conventional thyroidectomy, MIT: minimal invasive thyroidectomy

The distribution of postoperative histopathological diagnosis was as follows: 61 multinodular goiter (\%44), 8 follicular adenoma (6\%), 68 differentiated thyroid carcinoma (50\%). In MNG group, 25 of 61 patients (40\%) underwent minimally invasive surgery, and in 25 of 68 carcinoma patients (37\%) had a minimally invasive procedure (Table 2). In minimally invasive surgery group, 25 out of 53 (47\%) patients had a malignant pathology.

Table 2. Intraoperative and postoperative findings. Wong-Baker FACES Scale and VAS scale ranges from 0 to 10, 0 having no pain and 10 unbearable for Wong-Baker Scale; 0 not satisfied et al and 10 most satisfied for Visual Analogue Scale).

\begin{tabular}{|l|c|c|c|}
\hline & CT group & MIT group & p value \\
\hline Operative Time (minutes) & $59 \pm 22$ & $60 \pm 21$ & 0,821 \\
\hline Blood Loss (milliliters) & $70 \pm 42$ & $26 \pm 17$ & $<0,001$ \\
\hline Postoperative Pain (Wong-Baker FACES Scale) & 5,1 & 2,3 & $<0,001$ \\
\hline Cosmetic Outcome (VAS) & 8,1 & 9,7 & $<0,001$ \\
\hline Drain Usage (without/ with drain) & $2 / 82$ & $12 / 41$ & $<0,001$ \\
\hline
\end{tabular}

CT: conventional thyroidectomy, MIT: minimal invasive thyroidectomy

Operative time was 59 minutes for conventional thyroidectomy and 60 minutes for minimally invasive surgery group. Figure 1 shows the operative time and the change of duration through the years. Decrease in operative time is seen on the table and it was due to learning curve [9] and usage of LigaSure ${ }^{\mathrm{TM}}$ in surgery.

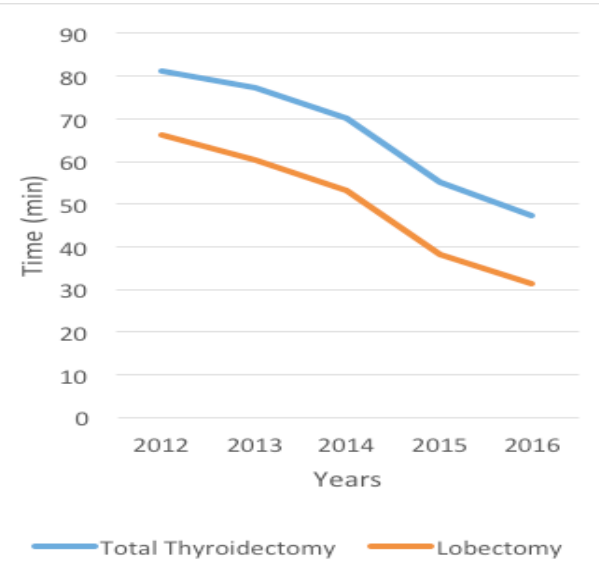

Figure 1. Mean operative time change by years. (Note that sealing device was seldomly used in 2014 and became routinely used after 2015.) 
Average blood loss were $70 \mathrm{ml}$ for conventional group and $26 \mathrm{ml}$ for minimally invasive group (Table 1). The difference between conventional and minimally invasive surgery group was statistically significant $(p<0.001)$.

Overall, in 123 patients, closed suction or open drains were used; in 14 patients, no drainage was needed. 12 of these 14 patients belonged to minimally invasive group (Table 1).

No conversion from minimally invasive video assisted thyroidectomy to conventional approach was required in our experience.

Average postoperative pain scale was 5.1 and 2.3 for conventional group and minimally invasive group respectively. Statistically, the difference between the two groups was significant $(p<0.001)$

Overall, 5 patients experienced transient hypocalcemia (3,6\%), 4 had permanent hypocalcemia (2.9\%), 2 had unilateral vocal cord paralysis (1.5\%), no patients experienced permanent bilateral vocal cord paralysis. 1 case had a postoperative hematoma (0.7\%), 1 10-year-old patient had an esophagus laceration manifested by saliva drainage to the suction drain and necessitated a surgical exploration (Table 3).

Table 3. Complications

\begin{tabular}{|c|c|c|c|c|c|}
\hline & CT group & MIT group & Total & p value \\
\hline Complications & $\begin{array}{c}\text { Transient } \\
\text { Hypocalcemia }\end{array}$ & 4 & 1 & $5(3,6 \%)$ & 0,276 \\
\cline { 2 - 5 } & $\begin{array}{c}\text { Permanent } \\
\text { Hypocalcemia } \\
\text { Vocal cord } \\
\text { paralysis } \\
\text { Hematoma } \\
\begin{array}{c}\text { Esophagus } \\
\text { Laceration }\end{array}\end{array}$ & 1 & 1 & $4(\% 3)$ & $2(2 \%)$ \\
\hline
\end{tabular}

CT: conventional thyroidectomy, MIT: minimal invasive thyroidectomy

Cosmetic outcome scores after conventional surgery group was 8.1; whereas the minimally invasive group scored 9.7. The difference between the conventional and minimally invasive surgery group was statistically significant $(p<0.001)$.

\section{DISCUSSION}

Thyroid surgery is a historical entity and its history spans more than a millennium, dating as back as Abu Al-Qasim with his first goiter excision recorded in Al-Tasrif in 952. However, due to life threatening bleeding and high perioperative and postoperative complications, thyroid surgery was feared by surgeons and patients, and nearly prohibited for centuries. It was Theodor Billroth and Theodor Kocher, the fathers of modern thyroid surgery, who changed mortality rates as high as $\% 75$, to less than $\% 1$ [10] . Theodor Kocher later won the Nobel Prize in 1909 for his work on thyroid surgery and thyroid metabolism and he was the first surgeon to be awarded. The main reason behind the increased safety and efficacy of the operations was better understanding of the anatomy, meticulous hemostasis, respect for the surrounding vital structures, and the emerging metabolic control of thyroid and parathyroid system [11].

However, the thyroid surgery is far from mastered; better visualization of thyroid vessels and recurrent the pursuit for the excellence is in progress, and laryngeal nerves [12-14]. surgeons all around the world and from different specialties still try to minimize operative complications, postoperative morbidities and better the cosmetic results. Among the modern procedures, robotic transaxillary and transvestibular thyroidectomies are being studied and publicized which eliminates a visible neck scar and improves the operative field. But they need an expensive foundation which most hospitals around the globe is missing and involve a learning curve. But before all this robotic, futuristic era; surgical minds were still searching for this cosmetic problem and were trying to improve the surgical visualization. With robotic thyroid surgery still progressing, many centers with conventional surgical setups are suitable for minimally invasive procedures and with little added instruments to their armamentaria, the surgeries can be performed without more effort. In the literature, minimally invasive thyroidectomies are favored for their lower postoperative pain, higher cosmetic outcomes and equal surgical safety and 
Average blood loss during the surgery was significantly lower for both total thyroidectomy and lobectomy in minimally invasive thyroidectomy group than conventional group in our study. In the literature, blood loss data was reported seldomly. The studies that examined the difference between conventional and minimally invasive video assisted thyroidectomy with regard to blood loss found no statistical difference between the groups $[12,15$, 16]. The difference could be partly due to better developing surgical planes by better visualization and partly due to meticulous hemostasis for the endoscope not to be soiled. This in turn, could result in less drain application. Indeed, our results show that minimally invasive thyroidectomy is more likely to end up without a drain than conventional one.

In addition, one of the main reasons for minimally invasive thyroidectomy like any other minimally invasive surgery is lower postoperative pain. Micoli et al., Bellantone et al. Lombardi et al., El-Labhan et al. examined this phenomenon and found that minimally invasive thyroidectomy results in significantly lower postoperative pain after 24 hours $[2,12$, 17]. Our findings support the literature on postoperative pain. The main reason behind this is less tissue disruption provided by small incision and better surgical visualization, thus less inflammation; resulting in less postoperative pain. Micoli et al. also found that serum TGF- $\beta$ levels and Visual Analogue Score for pain is inversely related and could be used to evaluate pain following minimally invasive thyroidectomy [18].

Considering that age and sex profile of the patients undergoing thyroidectomy, cosmetic concerns are somewhat more pronounced. In the literature, minimally invasive thyroidectomy results in better cosmetic outcomes $[2,3,8]$, and our findings are consistent with the literature.

All new surgical techniques must be at least as safe as the previous ones in order to be implemented in clinical use. It is even more pronounced when a new technique is proposed alternative to centuries old one such as thyroidectomy. In our case, different studies reveal that minimally invasive thyroidectomy results in statistically similar postoperative complications such as hypocalcemia, vocal cord paralysis $[2,14,16,17]$. When our study is concerned, although minimally invasive group has less postoperative complications; no statistical difference in occurrence of hypocalcemia and vocal cord paralysis between the conventional group and minimally invasive group were seen. Our findings correspond with the equal safety of minimally invasive thyroidectomy versus conventional thyroidectomy described in the literature.

Minimally invasive thyroid surgery is actually not a new concept; however, surgeons around are reluctant to adopt this new technique. The reasons behind this reluctance is fear of change, the idea that minimally invasive techniques require expensive set up of instruments, the learning curve that every other surgery, especially endoscopic ones require. All that the minimally invasive video assisted techniques essentially require is an endoscopy system and an endoscope. The endoscope could be 5 $\mathrm{mm}$ 0-degree or 30-degree if the surgeon is a laparoscopy-performer. Or it could be standard $4 \mathrm{~mm}$ endoscopic sinus surgery endoscopes, 0 or 30 -degree. All other instruments like aspirator-elevator and forceps are optional. Provided that most operating theatres have an endovision system, it actually requires no further setup.

\section{CONQUSION}

Our findings are consistent with the literature about the efficacy and safety of the minimally invasive thyroid surgery and this single center experience supports the use of minimally invasive thyroidectomy even in patients with well differentiated carcinomas. Further studies with more patients and longer surveillance are required to compare this technique with centuries old conventional one.

\section{STATEMENT of ETHICS}

All the subjects included in this study have given their written informed consents. This study was approved by Ethics Board of Hacettepe University (KA-130043).

\section{ACKNOWLEDGEMENT}

We would like to thank all the medical personnel who made this study possible.

\section{CONFUCTS of INTEREST}

The authors have no conflicts of interest to declare. 
[1]Miccoli P, Berti P, Conte M, et al. Minimally invasive surgery for thyroid small nodules: preliminary report. J Endocrinol Invest 1999; 22(11): 849-51.

[2]Miccoli P, Berti P, Raffaelli M, et al. Comparison between minimally invasive video-assisted thyroidectomy and conventional thyroidectomy: a prospective randomized study. Surgery 2001; 130(6): 1039-43.

[3]Lombardi CP, Raffaelli M, Princi P, et al. Video-assisted thyroidectomy: report of a 7-year experience in Rome. Langenbecks Arch Surg 2006; 391(3): 174-77.

[4]Terris DJ, Seybt MW, Elchoufi M, et al. Cosmetic thyroid surgery: defining the essential principles. Laryngoscope 2007; 117(7): 1168-72.

[5]Terris DJ, Angelos P, Steward DL, et al. Minimally invasive video-assisted thyroidectomy: a multi-institutional North American experience. Arch Otolaryngol Head Neck Surg 2008; 134(1): 81-84.

[6]Henry JF. Minimally invasive thyroid and parathyroid surgery is not a question of length of the incision. Langenbecks Arch Surg 2008; 393(5): 621-26.

[7]Terris DJ and Seybt MW. Classification system for minimally invasive thyroid surgery. ORL J Otorhinolaryngol Relat Spec 2008; 70(5): 287-291.

[8]Linos D. Minimally invasive thyroidectomy: a comprehensive appraisal of existing techniques. Surgery 2011; 150(1): 17-24.

[9]Pons Y, Verillaud B, Blancal JP, et al. Minimally invasive video-assisted thyroidectomy: Learning curve in terms of mean operative time and conversion and complication rates. Head Neck 2013; 35(8): 1078-82.
[10]Chiesa F. The 100 years Anniversary of the Nobel Prize Award winner Emil Theodor Kocher, a brilliant far-sighted surgeon. Acta Otorhinolaryngol Ital 2009; 29(6): 289.

[11]Billroth T. Die allgemeine chirurgische Pathologie und Therapie. 1871: Reimer.

[12]Bellantone R, Lombardi CP, Bossola M, et al. Video-assisted vs conventional thyroid lobectomy: a randomized trial. Arch Surg 2002; 137(3): 301-304; discussion 305.

[13]Terris DJ and Chin E. Clinical implementation of endoscopic thyroidectomy in selected patients. Laryngoscope 2006; 116(10): 1745-48.

[14]Scerrino G, et al., Minimally Invasive Video-Assisted Thyroidectomy: Analysis of Complications From a Systematic Review. Surg Innov 2019; 26(3): 381-87.

[15]Chao TC, Lin JD, and Chen MF. Video-assisted open thyroid lobectomy through a small incision. Surg Laparosc Endosc Percutan Tech 2004; 14(1): 15-19.

[16]El-Labban GM. Minimally invasive video-assisted thyroidectomy versus conventional thyroidectomy: A single-blinded, randomized controlled clinical trial. J Minim Access Surg 2009; 5(4): 97-102.

[17]Lombardi CP, Raffaelli M, Princi P, et al. Safety of video-assisted thyroidectomy versus conventional surgery. Head Neck 2005; 27(1): 58-64.

[18]Miccoli P, Rago R, Massi M, et al. Standard versus video-assisted thyroidectomy: objective postoperative pain evaluation. Surg Endosc 2010; 24(10): 2415-17. 